\title{
Septic Shock and the Heart
}

\author{
Pietro Bertini ${ }^{1}$ (1) $\cdot$ Fabio Guarracino ${ }^{1}$
}

Published online: 21 March 2019

(C) Springer Science+Business Media, LLC, part of Springer Nature 2019

\begin{abstract}
Purpose of Review The aim of this review is to analyze the cardiovascular pathophysiology of septic shock. Using visual representations of a left ventricular cycle in the pressure/volume plane, we describe hemodynamic derangement occurring in septic shock and subsequent changes at each step of treatment allowing a rapid understanding of complex alterations.

Recent Findings Acute circulatory failure during sepsis has to be counterbalanced rapidly and appropriately. The most recent guidelines rely on volume expansion, vasoactive, and inotropic support but underlining patients' pathophysiology is often undetermined.

Summary Diagnosis of the hemodynamic substrate needs to be carried out thoroughly, using echocardiography, now commonplace for the intensivist. A pathophysiological approach as we describe might help to understand complicated patterns allowing updates during resuscitation steps.

Finally, in our beliefs, clinicians should address septic shock resuscitation using a tailored approach and specific protocols, but their applicability needs to be investigated.
\end{abstract}

Keywords Pressure-volume loops · Ventriculo-arterial coupling · Sepsis · Septic shock · Hemodynamics · Pathophysiology · Critical care

$\begin{array}{ll}\text { Abbreviations } \\ \text { MAP } & \text { Mean arterial pressure } \\ \text { VE } & \text { Volume expansion } \\ \text { TPR } & \text { Total peripheral resistance } \\ \text { CVP } & \text { Central venous pressure } \\ \text { PAOP } & \text { Pulmonary artery occlusion pressure } \\ \text { CO } & \text { Cardiac output } \\ \text { Pms } & \text { Mean systemic filling pressure } \\ \text { Pmsa } & \text { Mean systemic filling pressure analogue } \\ \text { FHM } & \text { Functional hemodynamic monitoring } \\ \text { PPV } & \text { Pulse pressure variation } \\ \text { SVV } & \text { Stroke volume variation } \\ E_{\mathrm{dyn}} & \text { Dynamic elastance } \\ \text { Ea } & \text { Effective arterial elastance }\end{array}$

This article is part of the Topical Collection on Critical Care Anesthesia

Pietro Bertini

pietro.bertini@gmail.com

1 Cardiothoracic and Vascular Anaesthesia and Intensive Care, Department of Anaesthesia and Critical Care Medicine, Azienda Ospedaliero Universitaria Pisana, Via Paradisa n, 2, 56124 Pisa, Italy
$\mathrm{ScVO}_{2} \quad$ Central venous oxygen saturation

PVL Pressure-volume loop

Ees Left ventricular elastance

ESPVR End-systolic pressure-volume relationship

EDPVR End-dyastolic pressure-volume relationship

SBP Systolic blood pressure

ESP End-systolic pressure

EDP End-dyastolic pressure

EDV End-dyastolic volume

SV Stroke volume

VAC Ventriculo-arterial coupling

HR Heart rate

$\mathrm{MVO}_{2} \quad$ Myocardial oxygen consumption

PVA Pressure-volume area

SW Stroke work

PE Potential energy

RV Right ventricular

IABP Intra-aortic ballon pump

ECMO Extracorporeal membrane oxygenation

VA Veno-arterial (ECMO)

VV Veno-venous (ECMO)

LVAD Left ventricular assist device

MRI Magnetic resonance imaging 


\section{Introduction}

The management of acute circulatory failure during septic shock is difficult and must be carried out rapidly and appropriately.

In 2016, the definitions of sepsis and septic shock were reviewed and updated in a consensus conference $[1 \bullet \bullet]$. The term "severe sepsis" was eliminated and replaced with "sepsis," which is now considered life-threatening organ dysfunction caused by a deregulated response to infection. Septic shock is now considered a subset of sepsis in which mortality is increased and associated with circulatory, cellular, and metabolic abnormalities.

These definitions, although more comprehensive and simple, did not introduce profound changes in the characterization of circulatory dysfunction, which is simply defined as hypotension after adequate fluid resuscitation or the need for vasopressors to maintain a mean arterial pressure (MAP) equal to or above $65 \mathrm{mmHg}$ and a lactate level above $2 \mathrm{mmol} / \mathrm{L}$. Nevertheless, the definitions emphasize the main role of hemodynamic derangement relative to poor outcomes and mortality.

In the most recent guidelines, volume expansion (VE) is recommended as the first intervention to treat hemodynamic impairment by increasing the MAP, which is the primary endpoint of sepsis resuscitation; vasoactive agents and inotropes can be considered if the MAP remains low [2]. Unfortunately, this approach fails to address the pathophysiological state of a patient suffering from septic shock. For this reason, only 50\% of patients are volume responders in terms of MAP increases, and if vasoactive agents or inotropes are unable to further restore MAP, a patient's outcome is unfavorable.

Despite the efforts of such guidelines to propose new hemodynamic monitoring techniques to properly address resuscitation, they are still insufficient in terms of the action of fluids, vasopressors, or inotropes because they do not completely appreciate the complexity of the physiopathological characterization of hemodynamic dysregulation.

VE is considered beneficial, relatively unharmful, and reversible compared to vasoactive agents or inotropic therapy, and its action relies on Starling's law of the heart in subjects with sufficient preload reserve. VE is advised because a significant portion of patients exhibits the capacity for an improved MAP if fluid resuscitation is applied, but it is unclear if fluid application, even if the MAP is restored, will have consequences. In fact, excessive fluid administration is associated with increased mortality in septic shock patients [3].

Vasoconstrictors, mainly norepinephrine and vasopressin, are applied to counterbalance drops in total peripheral resistance (TPR), which often occur in septic shock due to the vasoactive action of mediators liberated during the initial phase of sepsis. Importantly, Cariou et al. demonstrated how the adrenergic response is blunted during the early and late phases of septic shock [4], which could be a cause of the ineffective application of norepinephrine.

Inotropic support is considered the last line of treatment according to guidelines and is justifiable if neither VE nor vasoactive agents can increase the MAP because contractility may be impaired and thus require restoration.

In patients who are unresponsive to this algorithm of treatment, a more thorough understanding of the cardiovascular pathophysiology underlying septic shock is needed.

\section{Diagnosis and Evaluation}

The diagnosis and evaluation of patients with septic shock are complicated and often carried out retrospectively. According to the guidelines, however, the hemodynamic instability that occurs with sepsis is easy to recognize because it simply involves a MAP reduction below $65 \mathrm{mmHg}$. Unfortunately, hemodynamic derangement in septic shock patients does not always present with the same features, and the diagnosis implies a solid understanding of cardiovascular physiology.

Since VE is the first-line therapy in the algorithm of treatment in septic shock resuscitation, the ability to early recognize the capacity of the cardiovascular system to sustain a fluid load is essential [5].

Classical hemodynamic monitoring techniques rely on static pressures, such as central venous pressure (CVP) or pulmonary artery occlusion pressure (PAOP), but the historical assimilation to preload reserve with these measurements has often been misguiding and can lead to confusion [6].

The Guytonian model of circulation [7] offers an interesting point of view as it focuses on the blood flow back to the heart from the venous system, referred to as venous return, as one of the determinants of cardiac output (CO) and fluid responsiveness. Venous return is the result of the differences between the mean systemic filling pressure (Pms) and CVP. VE increases Pms. For CO to increase accordingly, venous return should also increase, or the resistance to that flow should decrease. In fluid responders, $\mathrm{CO}$ increases much more with respect to small $\mathrm{CVP}$ increments. In non-responders, CVP increases equally to Pms. Such a CVP increase is the stopping point for fluid administration. The universal value of this model is controversial because it neglects the left heart and pulmonary circulation, but its clinical utility has been demonstrated [8]. Moreover, surrogates of Pms, such as the mean systemic filling pressure analogue (Pmsa), have allowed Guytonian physiological bedside monitoring [9].

Functional hemodynamic monitoring (FHM) allows an understanding of the underlying pathophysiological substrate by analyzing the hemodynamic response to a fixed intervention and predicting responses to future treatment. Based on the dynamic indexes of preload, the FHM concept has expanded relative to the investigation of fluid responsiveness, arterial load, and microcirculation [10]. 
In particular, FHM uses a volume challenge or a passive leg raise maneuver combined with measurements of $\mathrm{CO}$ changes during positive pressure ventilation to detect fluid responsiveness using dynamic indexes of preload. Michard recognized how arterial pulse pressure variation (PPV) during the respiratory cycle could be used to detect fluid responsiveness and discovered that PPV significantly reduced after VE in fluid responders compared to non-responders [11]. Subsequently, other dynamic indexes were introduced with the same purpose, including stroke volume variation (SVV) [12], pleth variability, aortic flow variability, vena cava diameter change, and internal jugular vein distensibility [13]. Despite their increasing use, these indexes share the same limitation of poor reliability in spontaneously breathing patients or in the presence of arrhythmia, acute cor pulmonale, or extensive vasoconstriction.

Arterial load variation relative to VE has recently been investigated as a measurement of effective arterial elastances (Ea) [14 $]$ and dynamic arterial elastances $\left(E_{\text {dyn }}\right)$ [15] to compensate for dynamic indexes in cases of increased arterial tension, such as in vasoactive therapy [16].

Recently, microcirculation monitoring at the bedside has become feasible, and hemodynamic coherence, or the agreement between micro- and macro-circulation, is an interesting topic in research that warrants expansion [17].

As very few studies have investigated the superiority of one technique over the others to address the subsequent steps in septic shock resuscitation, vasoactive and inotropic support, guidelines and protocols tend to rely on traditional MAP to monitor therapeutic success. In fact, a recent meta-analysis of large-scale studies declared that the early goal-directed therapy protocol of Rivers [18] based on central venous oxygen saturation $\left(\mathrm{ScVO}_{2}\right)$ measurement is not superior to the standard practice of targeting CVP $>8 \mathrm{mmHg}$ and MAP $>$ $65 \mathrm{mmHg}$ to improve patient outcomes [19].

\section{A Pathophysiological Representation}

The representation of heart dynamics in the pressure-volume plane allows an appreciation of the four phases of the cardiac cycle in a single-beat pressure-volume loop (PVL): filling, isovolumetric contraction, ejection, and isovolumetric relaxation.

Every loop can also be defined by two boundaries: the endsystolic pressure-volume relationship (ESPVR), which is mostly linear and defined by a slope called ventricular elastance (Ees) and a volume axis intercept V0, and the end diastolic pressure-volume relationship (EDPVR), which is curvilinear and well described by acknowledged functions. ESPVR and EDPVR represent changes in contractility and the diastolic properties of the ventricle, respectively.
The position and shape of the PVL depend on preload and afterload.

Preload can be defined by end-diastolic pressure (EDP) and volume (EDV) at the heart level. Afterload is much more influenced by arterial characteristics that oppose left ventricular ejection.

Afterload can be represented by effective arterial elastance $(\mathrm{Ea})$, which is the slope of a line intersecting with end diastolic volume on the volume axis and the end-systolic pressure-volume point on the PVL.

End-systolic pressure (ESP) is defined as the pressure at the end of the ejection phase and has been a subject of debate because end systole cannot be identified as the end of antegrade flow from the aortic valve in several situations, ranging from a very early termination of flow as in aortic regurgitation to a very delayed cessation of flow in the case of counterpulsation application. One of the most accepted surrogates for ESP is dicrotic notch pressure, which is considered an expression of practical sense and clinical applicability [14•, 20-22]. Other validated methods have also been described, including peak aortic systolic pressure, systolic blood pressure (SBP), MAP, $\frac{2 \cdot \mathrm{SBP}-\mathrm{DBP}}{3}$ and SBP·0.9 [14•, 23-30].

Ea can be therefore calculated as the ratio of ESP to stroke volume (SV).

Importantly, the ratio between $\mathrm{Ea}$ and Ees, called ventriculo-arterial coupling (VAC), has been used as a surrogate of the mechanical efficiency of the cardiovascular system and the heart and vessels in the systolic phase and has been investigated in the septic shock population [31•].

In fact, other properties and measurements such as $\mathrm{SV}$ or ejection fraction (EF) can be easily derived from VAC.

Diastolic properties of the ventricle are much more difficult to quantify, mostly because of the non-linearity of the EDPVR. Stiffness, in particular, is defined by the relationship $\mathrm{dP} / \mathrm{dV}$, the change in pressure for a given change in volume. Generally, diastolic stiffness increases as EDP increases in a non-linear fashion. For this reason, it is difficult to estimate in clinical practice. Left ventricular (LV) capacitance has been introduced to evaluate diastolic properties, with an index of V30 and a volume at EDP of $30 \mathrm{mmHg}$.

Furthermore, the pressure-volume plane offers the ability to investigate the determinants of myocardial oxygen consumption $\left(\mathrm{MVO}_{2}\right)$.

$\mathrm{MVO}_{2}$ is linearly related to the ventricular pressurevolume area (PVA), as the result of the sum of stroke work (SW) which is the area inside the PVL and the potential energy (PE), the residual area bounded by the ESPVR, the EDPVR, and the diastolic portions of the PVL. This latter surface represents the residual energy that is not used to produce external work. More PE represented with respect to SW corresponds to a more inefficient system, which means that more oxygen is wasted [32]. 


\section{Hemodynamics of Septic Shock at a Glance}

Historically, circulatory failure associated with sepsis has been assimilated to distributive shock, a hemodynamic characterization in which a healthy heart encounters less resistance to ejection due to peripheral vasodilation and tachycardia, leading to hyperdynamic state and a low MAP.

For this reason, intensivists tend to assume that septic shock patients have normal VAC and that hemodynamic derangement is the result of vasodilation due to sepsis. Monge et al. [15] assumed that septic shock patients have normal VAC in their analysis, but a recent study demonstrated that ventriculo-arterial uncoupling in septic shock is frequent $[31 \bullet]$.

In fact, in patients with elevated VAC, uncoupling could be the result of increased Ea, decreased Ees, or both. This is even more easy to accept if we consider sepsis-induced cardiomyopathy [33] and Takotsubo's syndrome related to the iatrogenic administration of high doses of vasoactive agents [34].

Lots of information can be appreciated by drawing a PVL in a two-dimensional plane, as in Fig. 1a.

Sepsis can cause a reduction in TPR due to the action of vasoactive mediators liberated in the early phases of septic shock, which can lead to an acute reduction in the circulating volume and tachycardia. Consequently, reductions in MAP and SV are apparent.

Despite the reduced volume ejected from the left ventricle, $\mathrm{CO}$ can be maintained or even increased by an increased heart rate (hyperdinamism). This has been historically defined as the "warm shock" phase.

As represented in Fig. 1b, we can observe huge reductions in SV and ESP (or MAP) and, surprisingly, a significant increase in Ea.

This may require further explanation: Ea is often mistaken or misinterpreted as TPR, but to be specific, given heart rate (HR):

$\mathrm{TPR}=\frac{\mathrm{MAP}-\mathrm{PVC}}{\mathrm{HR}} \mathrm{SV}$

If we neglect PVC and assimilate MAP as ESP, we find the following:

\section{$\mathrm{TPR} \cdot \mathrm{HR} \propto \frac{\mathrm{ESP}}{\mathrm{SV}}$}

However, $\frac{\mathrm{ESP}}{\mathrm{SV}}=\mathrm{Ea}$

Therefore, $\mathrm{Ea} \cong \mathrm{TRP} \cdot \mathrm{HR}$

For this reason, Ea depends on TPR and HR but is not equal to TPR. In fact, in situations where the TPR is blunted but the HR increases, Ea can be maintained or may even increase.
Moreover, with a reduction in ESP and a more profound reduction in $\mathrm{SV}$, as in this case, the equation is as follows:

$\mathrm{Ea}=\frac{\mathrm{ESP}}{\mathrm{SV}}$ is also satisfied.

As a result of this initial insult, VAC is deranged as Ees is maintained and Ea increases.

In addition, from an energetic point of view, the SW to PVA ratio is very reduced. Therefore, to maintain this hyperdynamic situation, the system expends more energy, which could lead to further derangement (Fig. 1j).

In this phase, the administration of fluids can be very helpful in increasing the MAP by increasing the volume, provided that contractility is preserved.

If the situation is not corrected, Ees, which reflects contractility, starts to decrease, leading to the representation in Fig. 1c.

Ees is reduced and SV is very reduced. CO starts to decrease, but Ea increases to maintain the MAP and the PVL is shifted to the right side of the plane until circulatory arrest.

\section{Therapy}

\section{The Guidelines}

The primary endpoint in septic shock resuscitation is to restore the MAP. To accomplish this, as correctly demonstrated in the guidelines, volume expansion is applied. Figure 1d shows what occurs after VE.

In a favorable situation after VE, SV increases, $\mathrm{CO}$ is maintained as HR decreases and most importantly, Ea is reduced, leading to VAC improvement and MAP restoration.

If the MAP is not restored, vasoconstrictive agents such as norepinephrine or vasopressin can be applied until the desired pressure is achieved.

Finally, if reasonable vasoconstriction has been achieved without an effect on the MAP, inotropic support using dopamine or dobutamine can be applied.

In a variable proportion of patients, Ees is depressed due to pre-existing cardiac conditions and/or sepsis cardiomyopathy. In these patients, applying more $\mathrm{VE}$ or vasoconstrictive agents can lead to further derangement (Fig. 1e).

The purpose of applying VE and the subsequent addition of vasopressors is to elicit the Frank-Starling mechanism and increase the EF and SV.

Unfortunately, analyses of such mechanical properties of the heart are rarely performed before starting the treatments. For this reason, predicting a patient's response to VE and determining which part of the starling curve reflects the patient's condition can be difficult.

In patients with depressed contractility, the application of inotropic agents to increase Ees may be considered before vasoactive medication or even before $\mathrm{VE}$ is completed 


\section{a - Normal PVL}

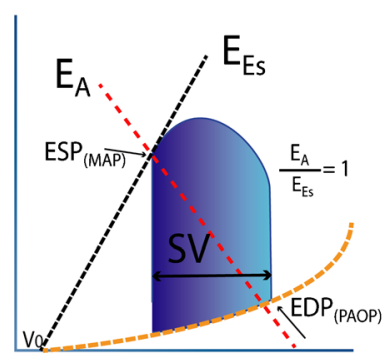

e- Negative Vasoactive effect $E_{E s} \downarrow$

$E_{A} \uparrow$

SV $\downarrow$

EDP $\uparrow$

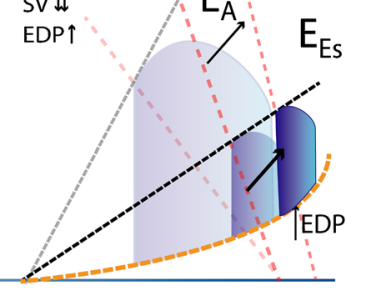

b-Septic Shock

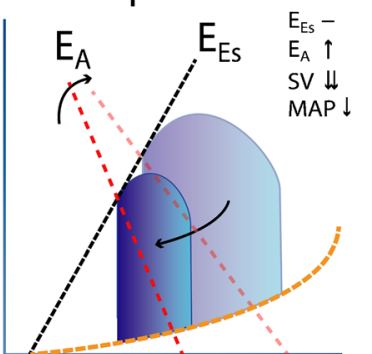

f - Inotropic support

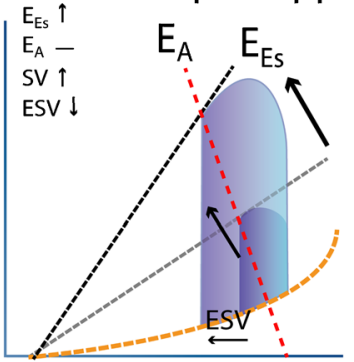

c - Septic Shock \& HF
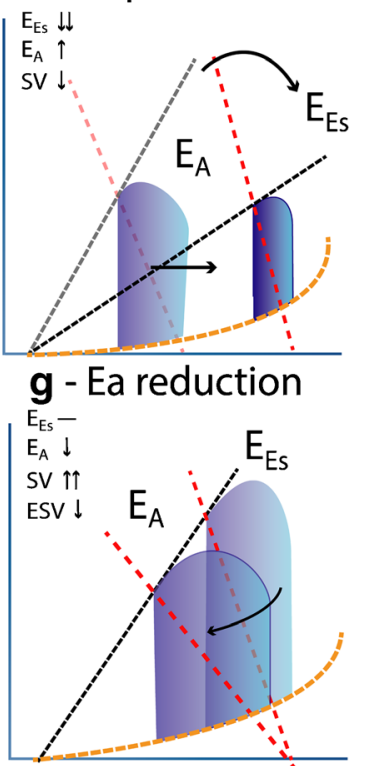

d - VE Resuscitation
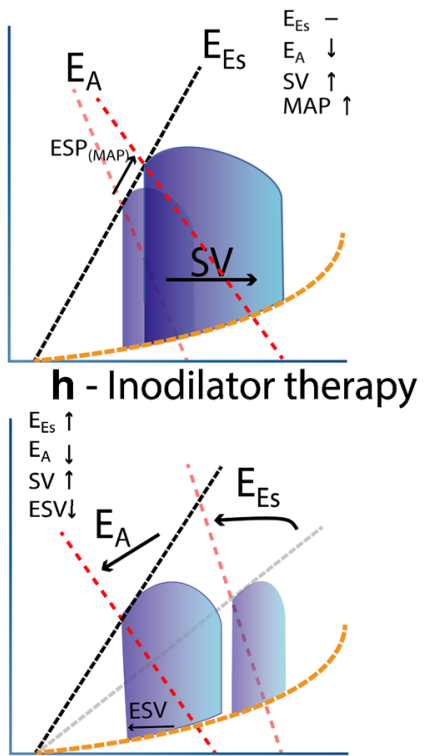
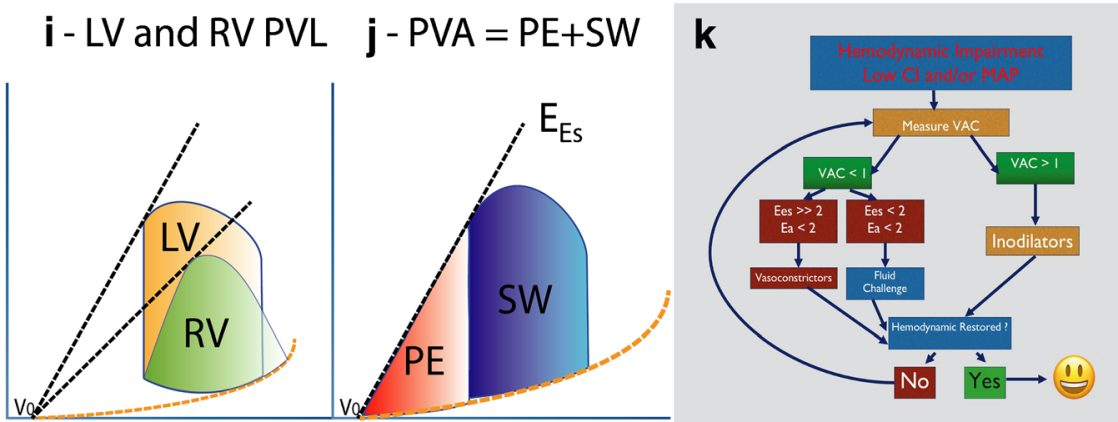

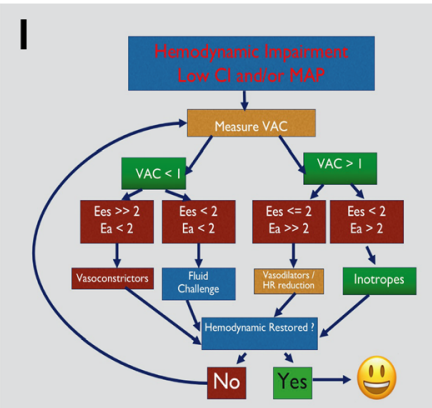

Fig. 1 a Normal PVL: Ea, effective arterial elastances, Ees, ventricular elastances; ESP, end-systolic pressure; EDP, end-diastolic pressure; V0, volume axis intercept; SV, stroke volume. b Septic shock can cause Ea increase by reduction in MAP and steeper decrease of SV. c Ees huge reduction leads to further hemodynamic derangement. $\mathbf{d}$ Effects of VE on PVL. e Ineffective application of VE or norepinephrine in patients with septic shock and reduction of contractility. $\mathbf{f}$ Effects of inotropic support in septic shock. $\mathbf{g}$ Effects of therapies aiming to reduce Ea in septic shock. h Effects of inodilator therapy on PVL. i LV, left ventricular PVL; RV,

(Fig. 1f). After VE, patients who do not exhibit MAP restoration due to the vasoplegic effect of the inflammatory response may either benefit from an increased afterload with subsequent sustained contractility or experience reduced contractility, for which vasoconstrictor application may reduce hemodynamic efficiency.

After Ees has been restored, Ea can still be elevated. To reduce $\mathrm{Ea}$, vasodilators, or other medications to reduce HR can be applied, as in Fig. 1g.

\section{Inodilators}

Inodilator therapy can be applied in patients experiencing septic shock to restore or prevent contractility right ventricular PVL. j PE, potential energy; SW, stroke work,; PVA= $\mathrm{PE}+\mathrm{SW}$. k Algorithm of treatment for hemodynamic instability in septic shock if inodilators are available; CI, cardiac index; MAP, mean arterial pressure; VAC, ventriculo-arterial coupling; Ea, arterial elastance; Ees, left ventricular elastance. I Algorithm of treatment for hemodynamic instability in septic shock if inodilators are not available; CI, cardiac index; MAP, mean arterial pressure; VAC, ventriculo-arterial coupling; Ea, arterial elastance; Ees, left ventricular elastance

derangement that leads to uncoupling. Although the application of drugs that could further reduce the MAP in hypotensive patients seems controversial, a recent study showed that compared to dobutamine, norepinephrine requirements were not higher in patients treated with levosimendan [35].

Further, Guarracino et al. demonstrated the beneficial effect of levosimendan on ventriculo-arterial coupling in ischemic cardiomyopathy [36].

While augmenting contractility, levosimendan affects the unloading of the left ventricle in the diastolic phase of the heart cycle. Therefore, it reduces Ea and increases SV and $\mathrm{CO}$, leading to quicker improvements in hemodynamics (Fig. 1h). 
Although most recent investigations have not clarified the beneficial effect of levosimendan over dobutamine in the treatment of septic shock [35,37], improvements seem to be evident in cellular metabolic alterations caused by sepsis [38]. Moreover, in a recent network meta-analysis, the authors concluded that levosimendan may have a superior beneficial effect on mortality [39•] compared to other drugs.

\section{Therapies to Control HR}

In a recent randomized trial, Morelli et al. investigated the role of short-acting beta blockers in septic shock patients [40]. Despite controversy, the use of beta blockers in the septic shock population has been shown to reduce mortality.

The application of beta blockers or other drugs that reduce HR, such as ivabradine, without affecting TPR leads to a dramatic reduction in Ea and prevents VAC derangement.

Ivabradine has been found to improve microvascular function in experimental sepsis [41], but further studies are necessary to determine whether its effects influence the macrocirculation similarly to beta blockers.

\section{Mechanical Support}

In refractory septic shock, mechanical support can be considered.

Intra-aortic balloon pump (IABP) was theorized to have successful applicability in septic shock to unload the left ventricle, but reports are very limited with the exception of animal studies [42].

Extracorporeal membrane oxygenation (ECMO) has become more important in the treatment of ARDS to restore oxygen delivery in patients suffering from respiratory insufficiency and are unresponsive to ventilation strategies [43]. $\mathrm{ECMO}$, in its veno-arterial (VA) configuration, can also provide mechanical support to the failing heart or in refractory cardiac shock following cardiac arrest [44].

There are several cases in which veno-venous (VV) ECMO was switched to VA ECMO or other hybrid techniques in circumstances of worsening septic shock. Although some have reported success [45], ECMO is generally not considered an optimal treatment for refractory septic shock. First, sepsis and septic shock may be associated with relevant coagulopathy. Heparin is usually given to maintain ECMO, which could lead to major bleeding, limiting its application in sepsis. Second, VA ECMO, in which blood is pumped from the right atrium to the arterial tree, has major hemodynamic effects, causing increases in EDP, MAP, and Ea that can lead to ventricular dilatation and a reduced $\mathrm{CO}$ [32]. If dilatation is extensive, unloading may be necessary to restore ejection and this can be achieved via IABP, LV venting, or other mechanical support such as the Impella device [46].
The application of other mechanical support strategies such as left ventricular assist devices (LVAD) in primary septic shock patients is rare. These devices, which allow left ventricle to aorta bypass or left atrium to aorta bypass, are usually employed in the context of cardiac surgery or transplantation. Nonetheless, these techniques increase perfusion and prevent ventricular overload and their application in refractory septic shock warrants investigation.

\section{The Right Ventricle and Ventricular Interdependence}

Right ventricular (RV) assessment in septic shock is crucial to prevent further hemodynamic derangement and implement the appropriate therapeutic approaches. Historically, RV dysfunction is underestimated until it becomes relevant [47].

$\mathrm{RV}$ insufficiency may be caused by the same vasoactive mediators involved in left ventricular dysfunction due to sepsis or by a mismatch in contractility and pulmonary arterial overload, which is not uncommon in ARDS, aggressive ventilation, increased left ventricular preload, or a combination of these. Regardless of the cause, increased mortality is evident in patients with RV failure and sepsis [48].

The pathophysiology of RV derangement in sepsis starts as systemic hypotension occurs, leading to reduced oxygen delivery and pulmonary vasoconstriction. As RV afterload increases, the RV increases in volume and pressure, which may lead to an interventricular septum shift to the left with resulting left ventricular diastolic impairment, further reducing the MAP and relative circulating volume.

In this situation of elicited ventricular interdependence, rapid strategies need to be implemented to restore RV function. These strategies usually involve targeted fluid administration, but too much VE can actually disrupt RV to pulmonary arterial coupling. Conversely, too little VE can cause insufficient upstream pressure to drive RV systole. Protective ventilator strategies can be instrumental in further reducing the RV afterload. Inotropes, inodilators, and nitric oxides can also be considered.

On the pressure-volume plane, the RV is represented as a loop, but it is slightly different and more triangular compared to the LV as the ejection of blood through the pulmonary valve may continue even when RV pressure falls due to reduced pulmonary artery impedance [49]. However, the pathophysiological concepts are essentially the same (Fig. 1i).

\section{PVL at the Bedside}

Real-time, accurate representations of the PVL are feasible when using invasive techniques such as conductance catheters in the cath-lab or the hybrid theater, but these approaches have 
poor reliability in other environments such as in the intensive care unit [50].

Echocardiography has become essential to intensivists attempting to assess critically ill patients at the bedside. With a focused hemodynamic approach, it is now possible to rapidly determine the causes of hemodynamic instability and monitor the therapeutic effects of interventions while screening for unexpected findings [51]. Moreover, using a pathophysiological approach, it is possible to measure Ees and Ea at the bedside using single-beat non-invasive methods [52••] and investigate cardiovascular mechanics without invasive procedures or modifications to a patient's loading conditions.

The pathophysiological model of the circulation has been applied to the RV as well [53, 54, 47], but only a small number of studies have investigated RV contractility and pulmonary elastance in critical care patients. RV-arterial coupling has been explored and calculated by different methods [55], which are essentially divided into volume-based methods, pressurebased method, and a mixture of the two. The advantage of volume-based methods relies on the precision of the imaging technique used to assess right ventricular volumes. Magnetic resonance imaging (MRI) is considered very accurate, but it is poorly applicable in the critical care setting. Therefore, 3D echocardiography and software reconstruction should be further investigated. Pressure-based techniques are essentially founded on pulmonary artery catheterization. Only one report, to our knowledge, describes a non-invasive bedside technique [56].

\section{Putting All Together and Where to Go From Here}

Ultimately, practical interventions need to be implemented to counteract the aggressive effects of sepsis on the cardiovascular system and clinicians need a protocol to rely on to apply their therapeutic efforts appropriately.

The relationship between the heart and the vasculature can be used to guide therapies during septic shock resuscitation but its clinical applicability needs to be investigated. Here, we advocate the use of an algorithm of treatment that incorporates the concepts described before. Figures $1 \mathrm{k}$ and 1 show two versions of the same protocol. The first protocol includes the use of inodilators, but the second version does not.

\section{Conclusions}

Investigations of heart function and cardiovascular interaction in septic shock are crucial, and a pathophysiological-oriented approach seems reasonable for the application of therapeutic efforts.
Although feasible at the bedside, such an approach implies comprehensive knowledge of the pathophysiology occurring in circulatory insufficiency, particularly during septic shock. Echocardiographic techniques are now commonplace in the intensive care unit, but this is not sufficient. Clinicians also need to apply functional and appropriate techniques and conduct a thorough evaluation of every single patient to avoid reliance on hemodynamic characterization. Whether this focused and personalized approach will improve patient outcomes remains to be seen.

\section{Compliance With Ethical Standards}

Conflict of Interest Pietro Bertini and Fabio Guarracino declare they have no conflict of interest.

Human and Animal Rights and Informed Consent This article does not contain any studies with human or animal subjects performed by any of the authors.

\section{References}

Papers of particular interest, published recently, have been highlighted as:

- Of importance

-• Of major importance

1.• Singer M, Deutschman CS, Seymour CW, Shankar-Hari M, Annane D, Bauer M, et al. The third international consensus definitions for sepsis and septic shock (Sepsis-3). JAMA. 2016;315(8): 801-10 Surviving sepsis campaign guidelines the worldwide accepted guidelines to treat sepsis and septic shock.

2. Cecconi M, De Backer D, Antonelli M, Beale R, Bakker J, Hofer C, et al. Consensus on circulatory shock and hemodynamic monitoring. Task force of the European Society of Intensive Care Medicine. Intensive Care Med. 2014;40(12):1795-815.

3. Marik PE, Linde-Zwirble WT, Bittner EA, Sahatjian J, Hansell D. Fluid administration in severe sepsis and septic shock, patterns and outcomes: an analysis of a large national database. Intensive Care Med. 2017;43(5):625-32.

4. Cariou A, Pinsky MR, Monchi M, Laurent I, Vinsonneau C, Chiche $\mathrm{JD}$, et al. Is myocardial adrenergic responsiveness depressed in human septic shock? Intensive Care Med. 2008;34(5):917-22.

5. Guerin L, Monnet X, Teboul JL. Monitoring volume and fluid responsiveness: from static to dynamic indicators. Best Pract Res Clin Anaesthesiol. 2013;27(2):177-85.

6. Berlin DA, Bakker J. Starling curves and central venous pressure. Crit Care. 2015;19:55.

7. Maas JJ, Pinsky MR, Geerts BF, de Wilde RB, Jansen JR. Estimation of mean systemic filling pressure in postoperative cardiac surgery patients with three methods. Intensive Care Med. 2012;38(9):1452-60.

8. Furst B. The heart: pressure-propulsion pump or organ of impedance? J Cardiothorac Vasc Anesth. 2015;29(6):1688-701.

9. Vos JJ, Kalmar AF, Hendriks HG, Bakker J, Scheeren TW. The effect of fluid resuscitation on the effective circulating volume in patients undergoing liver surgery: a post-hoc analysis of a randomized controlled trial. J Clin Monit Comput. 2017. 
10. Suess EM, Pinsky MR. Hemodynamic monitoring for the evaluation and treatment of shock: what is the current state of the art? Semin Respir Crit Care Med. 2015;36(6):890-8.

11. Michard F, Boussat S, Chemla D, Anguel N, Mercat A, Lecarpentier Y, et al. Relation between respiratory changes in arterial pulse pressure and fluid responsiveness in septic patients with acute circulatory failure. Am J Respir Crit Care Med. 2000;162(1): 134-8.

12. Reuter DA, Felbinger TW, Schmidt C, Kilger E, Goedje O, Lamm $\mathrm{P}$, et al. Stroke volume variations for assessment of cardiac responsiveness to volume loading in mechanically ventilated patients after cardiac surgery. Intensive Care Med. 2002;28(4):392-8.

13. Guarracino F, Ferro B, Forfori F, Bertini P, Magliacano L, Pinsky MR. Jugular vein distensibility predicts fluid responsiveness in septic patients. Crit Care. 2014;18(6):647.

14. Kelly RP, Ting CT, Yang TM, Liu CP, Maughan WL, Chang MS, et al. Effective arterial elastance as index of arterial vascular load in humans. Circulation. 1992;86(2):513-21 The first study that addressed ventriculo-arterial uncoupling in human septic shock.

15. Monge Garcia MI, Gil Cano A, Gracia Romero M. Dynamic arterial elastance to predict arterial pressure response to volume loading in preload-dependent patients. Crit Care. 2011;15(1):R15.

16. Monge Garcia MI, Saludes Orduna P, Cecconi M. Understanding arterial load. Intensive Care Med. 2016;42(10):1625-7.

17. Morelli A, Passariello M. Hemodynamic coherence in sepsis. Best Pract Res Clin Anaesthesiol. 2016;30(4):453-63.

18. Rivers E, Nguyen B, Havstad S, Ressler J, Muzzin A, Knoblich B, et al. Early goal-directed therapy in the treatment of severe sepsis and septic shock. N Engl J Med. 2001;345(19):1368-77.

19. Angus DC, Bailey M, Barnato AE, Bellomo R, CanterRR, Coats TJ, et al. PRISM Investigators. Early, goal-directed therapy for septic shock - a patient-level meta-analysis. N Engl J Med 2017;376(23):2223-2234. https://doi.org/10.1056/ NEJMoa1701380

20. Marchionni N, Fumagalli S, Baldereschi G, Di Bari M, Fantini F. Effective arterial elastance and the hemodynamic effects of intraaortic balloon counterpulsation in patients with coronary heart disease. Am Heart J. 1998;135(5 Pt 1):855-61.

21. Sunagawa K, Maughan WL, Sagawa K. Optimal arterial resistance for the maximal stroke work studied in isolated canine left ventricle. Circ Res. 1985;56(4):586-95.

22. Dahlgren G, Veintemilla F, Settergren G, Liska J. Left ventricular end-systolic pressure estimated from measurements in a peripheral artery. J Cardiothorac Vasc Anesth. 1991;5(6):551-3.

23. Scolletta S, Bodson L, Donadello K, Taccone FS, Devigili A, Vincent JL, et al. Assessment of left ventricular function by pulse wave analysis in critically ill patients. Intensive Care Med. 2013;39(6):1025-33.

24. Redfield MM, Jacobsen SJ, Borlaug BA, Rodeheffer RJ, Kass DA. Age- and gender-related ventricular-vascular stiffening: a community-based study. Circulation. 2005;112(15):2254-62.

25. Monge Garcia MI, Jian Z, Settels JJ, Hatib F, Cecconi M, Pinsky MR. Reliability of effective arterial elastance using peripheral arterial pressure as surrogate for left ventricular end-systolic pressure. J Clin Monit Comput. 2018.

26. Vizzardi E, Sciatti E, Bonadei I, D'Aloia A, Tartiere-Kesri L, Tartiere JM, et al. Effects of spironolactone on ventricular-arterial coupling in patients with chronic systolic heart failure and mild symptoms. Clin Res Cardiol. 2015;104(12):1078-87.

27. Monge Garcia MI, Guijo Gonzalez P, Gracia Romero M, Gil Cano A, Oscier C, Rhodes A, et al. Effects of fluid administration on arterial load in septic shock patients. Intensive Care Med. 2015;41(7):1247-55.

28. Chemla D, Antony I, Lecarpentier Y, Nitenberg A. Contribution of systemic vascular resistance and total arterial compliance to effective arterial elastance in humans. Am J Physiol Heart Circ Physiol. 2003;285(2):H614-20.

29. Najjar SS, Schulman SP, Gerstenblith G, Fleg JL, Kass DA, O'Connor F, et al. Age and gender affect ventricular-vascular coupling during aerobic exercise. J Am Coll Cardiol. 2004;44(3):6117.

30. Otsuki T, Maeda S, Iemitsu M, Saito Y, Tanimura Y, Ajisaka R, et al. Contribution of systemic arterial compliance and systemic vascular resistance to effective arterial elastance changes during exercise in humans. Acta Physiol (Oxf). 2006;188(1):15-20.

31. Guarracino F, Ferro B, Morelli A, Bertini P, Baldassarri R, Pinsky MR. Ventriculoarterial decoupling in human septic shock. Crit Care. 2014;18(2):R80 The first study that addressed ventriculo-arterial uncoupling in human septic shock.

32. Burkhoff D, Sayer G, Doshi D, Uriel N. Hemodynamics of mechanical circulatory support. J Am Coll Cardiol. 2015;66(23): 2663-74.

33. Sato R, Nasu M. A review of sepsis-induced cardiomyopathy. J Intensive Care. 2015;3:48.

34. Izumi Y. Drug-induced takotsubo cardiomyopathy. Heart Fail Clin. 2013;9(2):225-31 ix-x.

35. Bhattacharjee S, Soni KD, Maitra S, Baidya DK. Levosimendan does not provide mortality benefit over dobutamine in adult patients with septic shock: a meta-analysis of randomized controlled trials. J Clin Anesth. 2017;39:67-72.

36. Guarracino F, Cariello C, Danella A, Doroni L, Lapolla F, Stefani $\mathrm{M}$, et al. Effect of levosimendan on ventriculo-arterial coupling in patients with ischemic cardiomyopathy. Acta Anaesthesiol Scand. 2007;51(9):1217-24.

37. Landoni G, Lomivorotov VV, Alvaro G, Lobreglio R, Pisano A, Guarracino F, et al. Levosimendan for hemodynamic support after cardiac surgery. N Engl J Med. 2017;376(21):2021-31.

38. Hajjej Z, Meddeb B, Sellami W, Labbene I, Morelli A, Ferjani M. Effects of Levosimendan on cellular metabolic alterations in patients with septic shock: a randomized controlled pilot study. Shock. 2017;48:307-12.

39. Belletti A, Benedetto U, Biondi-Zoccai G, Leggieri C, Silvani P, Angelini GD, et al. The effect of vasoactive drugs on mortality in patients with severe sepsis and septic shock. A network metaanalysis of randomized trials. J Crit Care. 2017;37:91-8 The first study that addressed ventriculo-arterial uncoupling in human septic shock.

40. Morelli A, Ertmer C, Westphal M, Rehberg S, Kampmeier T, Ligges S, et al. Effect of heart rate control with esmolol on hemodynamic and clinical outcomes in patients with septic shock: a randomized clinical trial. JAMA. 2013;310(16):1683-91.

41. Miranda ML, Balarini MM, Balthazar DS, Paes LS, Santos MS, Bouskela E. Ivabradine attenuates the microcirculatory derangements evoked by experimental sepsis. Anesthesiology. 2017;126(1):140-9.

42. Solomon SB, Minneci PC, Deans KJ, Feng J, Eichacker PQ, Banks $\mathrm{SM}$, et al. Effects of intra-aortic balloon counterpulsation in a model of septic shock. Crit Care Med. 2009;37(1):7-18.

43. Abrams D, Brodie D. Extracorporeal membrane oxygenation for adult respiratory failure: 2017 update. Chest. 2017;152:639-49.

44. Kilic A, Shukrallah BN, Kilic A, Whitson BA. Initiation and management of adult veno-arterial extracorporeal life support. Ann Transl Med. 2017;5(4):67.

45. Park JI, Jung BH, Lee SG. Veno-arterial-venous hybrid mode of extracorporeal membrane oxygenation for acute respiratory distress syndrome combined with septic shock in a liver transplant patient: a case report. Transplant Proc. 2017;49(5):1192-5.

46. Kapur NK, Alkhouli M, DeMartini T, Faraz H, George Z, Goodwin $\mathrm{M}$, et al. Unloading the left ventricle before reperfusion in patients with anterior ST-segment elevation myocardial infarction: a pilot 
study using the Impella CP((R)). Circulation 2019;139(3):337-346. https://doi.org/10.1161/CIRCULATIONAHA.118.038269.

47. Pinsky MR. The right ventricle: interaction with the pulmonary circulation. Crit Care. 2016;20:266.

48. Chan CM, Klinger JR. The right ventricle in sepsis. Clin Chest Med. 2008;29(4):661-76 ix.

49. Greyson CR. The right ventricle and pulmonary circulation: basic concepts. Rev Esp Cardiol. 2010;63(1):81-95.

50. Clark JE, Marber MS. Advancements in pressure-volume catheter technology - stress remodelling after infarction. Exp Physiol. 2013;98(3):614-21.

51. Guarracino F, Bertini P. Perioperative haemodynamic management: is echocardiography the right tool? Curr Opin Crit Care. 2014;20(4):431-7.

52.• Chen CH, Fetics B, Nevo E, Rochitte CE, Chiou KR, Ding PA, et al. Noninvasive single-beat determination of left ventricular endsystolic elastance in humans. J Am Coll Cardiol. 2001;38(7):202834 The first study that addressed ventriculo-arterial uncoupling in human septic shock.
53. Guarracino F, Bertini P, Pinsky MR. Right Ventriculo-arterial coupling in the critically Ill. In: Vincent JL, editor. Annual update in intensive care and emergency medicine. Springer, Cham; 2017. https://doi.org/10.1007/978-3-319-51908-1_17

54. Bellofiore A, Chesler NC. Methods for measuring right ventricular function and hemodynamic coupling with the pulmonary vasculature. Ann Biomed Eng. 2013;41(7):1384-98.

55. Naeije R. Assessment of right ventricular function in pulmonary hypertension. Curr Hypertens Rep. 2015;17(5):35.

56. Bertini P, Guarracino F, Colosimo D, Gonnella S, Brizzi G, Mancino G, et al. Right ventricular-vascular coupling: a singlebeat noninvasive approach in the critically ill. Crit Care. 2017;21(Supp. 1):42.

Publisher's Note Springer Nature remains neutral with regard to jurisdictional claims in published maps and institutional affiliations. 